\title{
Impairment of Protease-Activated Receptor 2-Induced Relaxation of Aortas of Aged Spontaneously Hypertensive Rat
}

\author{
Makoto Ando," Takayuki Matsumoto," Shota Kobayashi, Maika Iguchi, Kumiko Taguchi, and \\ Tsuneo Kobayashi*
}

Department of Physiology and Morphology, Institute of Medicinal Chemistry, Hoshi University; 2-4-1 Ebara, Shinagawa-ku, Tokyo 142-8501, Japan.

Received December 7, 2017; accepted February 5, 2018

\begin{abstract}
Hypertension is one of the most prevalent diseases worldwide and can cause harmful complications within the vascular system. Further research on vascular responsiveness to different ligands and diverse receptors in various arteries is required to understand the mechanisms underlying the development of these vascular complications. Here, we investigated the vasorelaxant effect of the protease-activated receptor 2 (PAR2) agonist 2-furoyl-LIGRLO-amide (2-Fly) and two commonest agents, namely endothelium-dependent dilator acetylcholine (ACh) and endothelium-independent dilator sodium nitroprusside (SNP), on the thoracic aorta isolated from aged spontaneously hypertensive rats (SHR) (age, $52 \pm 1$ weeks). The effects of these agents were compared between aortas isolated from SHR and age-matched normotensive Wistar Kyoto (WKY) rats. Compared with the WKY group, in the SHR group, 2-Fly-induced relaxation was impaired, ACh-induced relaxation was slightly decreased at low concentrations, and SNP-induced relaxation was similar. In addition, 2-Fly-induced aortic relaxation was largely decreased by a PAR2 antagonist (FSLLRY), endothelial denudation, and a nitric oxide (NO) synthase inhibitor $N^{\mathrm{G}}$-nitro-L-arginine (L-NNA) but not by an Akt inhibitor. These results suggested that PAR2-induced relaxations of aortas of aged SHR was impaired, and this impaired aortic relaxation may be attributed to decreased NO bioavailability rather than altered NO sensitivity unrelated to the Akt activity.
\end{abstract}

Key words aorta; endothelium; hypertension; protease-activated receptor 2 (PAR2); relaxation

Vascular complications often occur systemically and arise in response to hypertension-associated pathologies, leading to increased mortality and morbidity rates in hypertensive patients worldwide. ${ }^{1)}$ A growing body of evidence suggests that an abnormal vascular tone, for example, augmented contraction and/or restricted relaxation that was induced by various ligands in hypertensive patients and animal models. ${ }^{2-4)}$ However, completely understanding vascular tone regulation under pathophysiological conditions is highly complex because the action of one ligand on a receptor may evoke different responses among different vessel types. Thus, a better understanding of the association between hypertension and vascular tone, as modulated by receptor activation, will help identify new therapeutic targets for preventing and treating hypertension-associated vascular complications.

Protease-activated receptor 2 (PAR2) is a member of the family of protease-activated receptors and is uniquely activated by proteolysis, PAR2 plays important roles in regulating diverse cellular processes during pathophysiological conditions such as atherosclerosis, diabetes, and hypertension. ${ }^{5-9)}$ In the vascular system, PAR2 activation can modulate the vascular tone. For example, PAR2 activation induced by the PAR2-activating peptide 2-furoyl-LIGRLO-amide (2-Fly) and endothelium-dependent relaxation induced by serine proteases occur by activating the endothelial nitric oxide synthase (eNOS) and eNOS-independent pathways in diverse vessels of many species. ${ }^{10-16)}$ Several studies compared PAR2-induced relaxation of vessels in healthy and diseased conditions such as diabetes and metabolic syndrome. ${ }^{10-12,17-19)}$ However,

\footnotetext{
\# These authors contributed equally to this work.

* To whom correspondence should be addressed. e-mail: tkoba@hoshi.ac.jp
}

knowledge regarding PAR2-induced responses in the vasculature during chronic hypertension is insufficient.

This study aimed to investigate whether PAR2-induced relaxation of aortas isolated from chronic hypertensive rats was altered. We used aortas isolated from aged spontaneously hypertensive rats (SHR) and age-matched, normotensive Wistar Kyoto (WKY) rats. We also compared aortic relaxation induced by two common dilatory agents, namely endothelium-dependent dilator acetylcholine (ACh) and endotheliumindependent dilator sodium nitroprusside (SNP, an NO donor), and investigated the mechanisms underlying PAR2-induced relaxation of aortas.

\section{MATERIALS AND METHODS}

Animals Four-week-old male SHR and WKY rats were obtained from Hoshino Laboratory Animals, Inc. (Ibaraki, Japan). All animals were housed under conditions of 12-h:12$\mathrm{h}$ light-dark cycles and were fed a standard chow diet with water ad libitum until they were $52 \pm 1$ weeks old. All animal experiments were approved by the Animal Care Committee of the Hoshi University. One week before euthanasia, systolic blood pressure (SBP) was measured by the tail-cuff method (BP-98A; Softron, Tokyo, Japan) as previously reported. ${ }^{20)}$

Isolation of Aortas and Functional Studies Surgical procedures were conducted under isoflurane-induced anesthesia, and vascular isometric forces were recorded in an organ bath, as previously reported. ${ }^{21,22)}$ After euthanasia, thoracic aortas were isolated and placed in ice-cold modified Krebs-Henseleit solution (KHS) that comprised $118.0 \mathrm{~mm}$ $\mathrm{NaCl}, 4.7 \mathrm{~mm} \mathrm{KCl}, 25.0 \mathrm{~mm} \mathrm{NaHCO}_{3}, 1.8 \mathrm{~mm} \mathrm{CaCl}_{2}, 1.2 \mathrm{~mm}$ 
$\mathrm{NaH}_{2} \mathrm{PO}_{4}, 1.2 \mathrm{~mm} \mathrm{MgSO}$, and $11.0 \mathrm{~mm}$ glucose. All connective tissues and fats were removed from the aortas. The aortas were then cut into 2-mm rings, placed in an organ bath filled with KHS, and continuously gassed with $95 \% \mathrm{O}_{2}, 5 \% \mathrm{CO}_{2}$ while maintaining the temperature at $37^{\circ} \mathrm{C}$. The aortic rings were stretched until an optimal resting tension of $2.75 \mathrm{~g}$ was achieved; they were then left to equilibrate for at least $45 \mathrm{~min}$ as previously reported. ${ }^{22}$ The aortic rings were precontracted with equieffective concentrations of phenylephrine (PE, Sigma-Aldrich, St. Louis, MO, U.S.A.), and concentration-relaxation curves were produced using 2-Fly $\left(10^{-10}-10^{-6} \mathrm{M}\right.$, Tocris Bioscience, Bristol, U.K.), ACh $\left(10^{-9}-10^{-5} \mathrm{M}\right.$, Daiichi-Sankyo Pharmaceuticals, Tokyo, Japan), or SNP $\left(10^{-10}-10^{-5} \mathrm{M}\right.$, Wako Pure Chemical Industries, Ltd., Osaka, Japan).

Concentration-relaxation curves for 2-Fly $\left(10^{-10}-10^{-6} \mathrm{M}\right)$ were obtained under the following conditions; endothelial denudation, treated with the PAR2 antagonist FSLLRY-NH $\left(10^{-6} \mathrm{M}\right.$; Tocris), the NOS inhibitor $N^{\mathrm{G}}$-nitro-L-arginine (L-NNA) $\left(10^{-4} \mathrm{M}\right.$; Sigma-Aldrich), or the Akt inhibitor $1 \mathrm{~L}-$ 6-hydroxymethyl-chiro-inositol $2[(R)$-2-O-methyl-3-O-octadecyl-sn-glycerocarbonate] $\left(10^{-6} \mathrm{M}\right.$; Calbiochem, San Diego, CA, U.S.A.) for $30 \mathrm{~min}$ prior to PE application. Endothelium denudation of the aortic ring was achieved by gently rubbing the lumen of the vessels with a pipette tip.

Data Analysis and Statistical Analysis Data are expressed as mean \pm standard error (S.E.). Relaxation responses are presented as percentages of the PE-induced precontraction value. $\mathrm{pD}_{2}$ was calculated as the negative logarithm of $\mathrm{EC}_{50}$ for each concentration-response curve. Concentration-response curves were fitted to all points between the lowest drug concentration and the maximal concentration at which the response from the tissue was elicited. For statistical analyses, Student's $t$-test was performed to compare between two groups and one-way ANOVA followed by Tukey's test was performed to compare among three or more groups. Statistical analyses of the concentration-response curves were performed using two-way ANOVA with repeated measures, followed by Bonferroni post hoc test. All data analysis was performed using GraphPad Prism (ver. 5.0 for Mac, San Diego, CA, U.S.A.). $p$ values of $<0.05$ were considered to be statistically significant.

\section{RESULTS}

General Parameters of Animals SBP was higher in the SHR group (SHR: $200 \pm 7 \mathrm{mmHg}, n=14$ ) than in the WKY group $(106 \pm 3 \mathrm{mmHg}, n=14) \quad(p<0.05)$. Body weight when killing the rats was lower in the SHR group $(460.7 \pm 6.6 \mathrm{~g}$, $n=14)$ than in the WKY group $(500.1 \pm 9.7 \mathrm{~g}, n=14)(p<0.05)$.

Relaxation Responses to 2-Fly, ACh, and SNP Once PE-induced contraction plateaued, 2-Fly $\left(10^{-10}-10^{-6} \mathrm{M}\right)$ was cumulatively added. The results are summarized in Figs. $1 \mathrm{~A}-\mathrm{C}$ and Table 1. Concentration-dependent relaxation of the aortic rings by 2-Fly was observed in both the groups, but relaxation was less reduced in the SHR group compared to that in the WKY group.

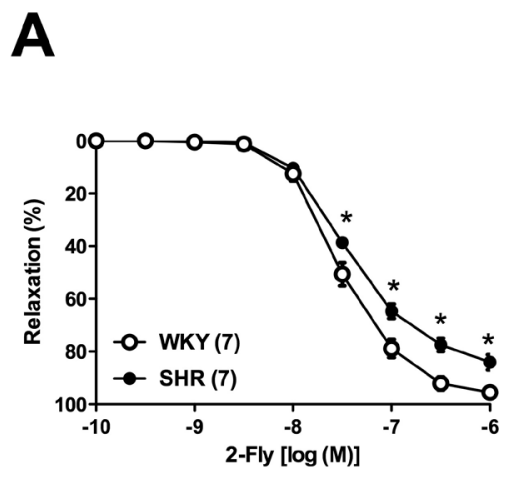

B
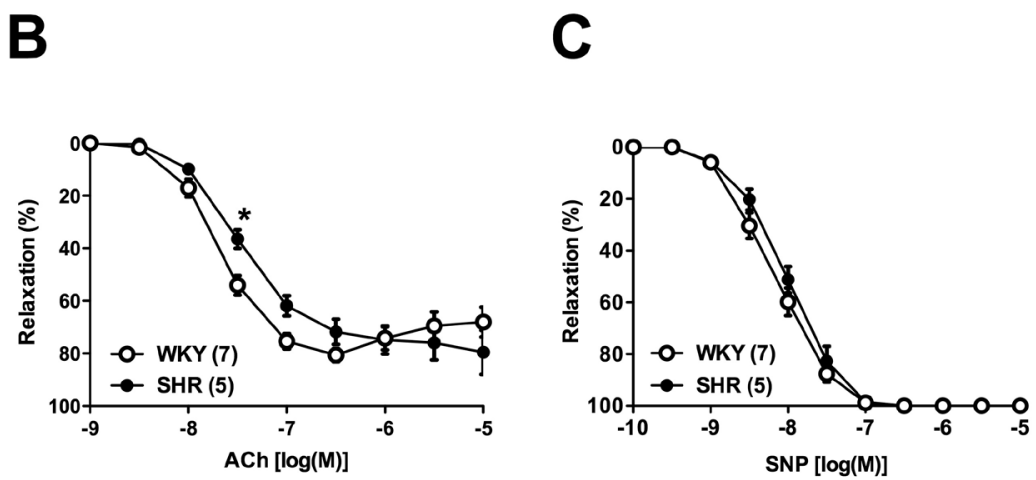

Fig. 1. Concentration-Response Curves for (A) 2-Fly-, (B) ACh-, and (C) SNP-Induced Relaxation of Aortic Rings Isolated from Aged SHRs and Age-Matched Normotensive WKY Rats

Data are expressed as mean \pm S.E. from five to seven experiments. Number of determinations $(n)$ is shown within parentheses. ${ }^{*} p<0.05$, SHR $v s$. WKY.

Table 1. Values of Maximal Relaxation $\left(E_{\max }\right)$ and $\mathrm{p} D_{2}$ in Concentration-Response Curves for 2-Fly, ACh, and SNP

\begin{tabular}{|c|c|c|c|c|c|}
\hline & & & 2-Fly & $\mathrm{ACh}$ & SNP \\
\hline \multirow{4}{*}{ Aorta } & \multirow{2}{*}{$E_{\max }$} & WKY & $95.6 \pm 1.8(7)$ & $81.8 \pm 2.8(7)$ & $100.0 \pm 0.0$ \\
\hline & & SHR & $84.2 \pm 2.9(7)^{*}$ & $81.6 \pm 7.5(5)$ & $100.0 \pm 0.0$ \\
\hline & \multirow[t]{2}{*}{$\mathrm{p} D_{2}$} & WKY & $7.46 \pm 0.06$ & $7.68 \pm 0.06(7)$ & $8.20 \pm 0.08$ \\
\hline & & SHR & $7.38 \pm 0.04$ & $7.45 \pm 0.06(5)^{*}$ & $8.05 \pm 0.09$ \\
\hline \multicolumn{6}{|c|}{$E_{\max }$ (concentration-response curve for 2-Fly) } \\
\hline & \multicolumn{2}{|r|}{ FSLLRY-NH ${ }_{2}$} & $\mathrm{EC}^{-}$ & L-NNA & Akt inhibitor \\
\hline WKY & \multirow{2}{*}{\multicolumn{2}{|c|}{$\begin{array}{l}29.0 \pm 6.9(3) \# \\
25.5 \pm 2.3(3) \dagger\end{array}$}} & $5.9 \pm 2.8(7) \#$ & $17.8 \pm 2.9(7) \#$ & $85.7 \pm 3.5(3)$ \\
\hline SHR & & & $11.0 \pm 3.2(6) \dagger$ & $4.9 \pm 2.1(7) \dagger$ & $68.4 \pm 7.5(3)$ \\
\hline
\end{tabular}

Values are given as means \pm S.E. Number of experiments is shown within parentheses. ${ }^{*} p<0.05 v s$. WKY (unpaired $t$-test), \#p<0.05 $v s$. untreated WKY, $\dagger p<0.05 v s$. untreated SHR (one-way ANOVA followed by Tukey's test). 
A

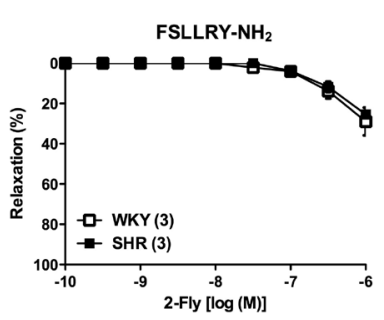

B

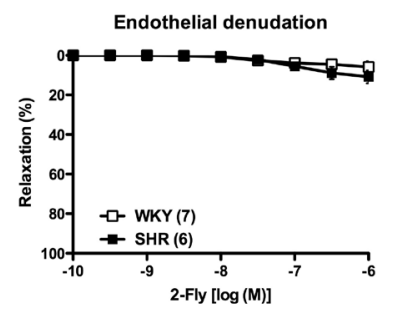

C

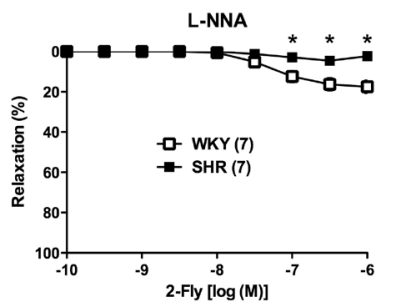

D

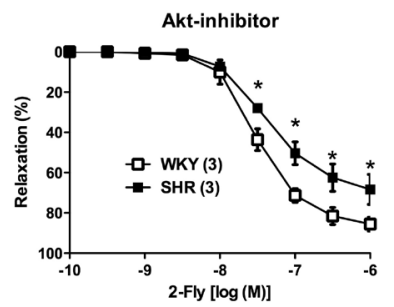

Fig. 2. Role of (A) PAR2, (B) Endothelium, (C) NOS and (D) Akt on 2-Fly-Induced Relaxation of Aortic Rings Isolated from Aged SHRs and AgeMatched Normotensive WKY Rats

Concentration-response curves for 2-Fly-induced relaxation in the presence of PAR2 antagonist FSLLRY-NH 2 (10 $\left.{ }^{-6} \mathrm{M}\right)(\mathrm{A})$, NOS inhibitor L-NNA (10 ${ }^{-4} \mathrm{M}$ ), or Akt inhibitor $\left(10^{-6} \mathrm{M}\right)$ in endothelium-intact aortas or endothelium-denuded aortas (B). Data are expressed as mean \pm S.E. from three to seven experiments. Number of determinations $(n)$ is shown within parentheses. $* p<0.05 v s$. corresponding WKY rats.

To confirm ligand specificity, we performed concentration-response curves for two common vasodilator ACh and SNP. The endothelium-dependent vasodilator $\mathrm{ACh}$ induced relaxation of the aorta (Fig. 1B), whereas the endotheliumindependent vasodilator SNP induced concentration-dependent relaxation of the aorta (Fig. 1C). ACh-induced relaxation of the aorta was slightly decreased at low concentrations in the SHR group compared with that in the WKY group (Fig. 1B); however, SNP-induced relaxation was similar between two groups (Fig. 1C).

Effect of PAR2 Antagonist, Endothelial Denudation, NOS Inhibitor, and Akt Inhibitor on 2-Fly-Induced Aortic Relaxation To investigate the possible mechanisms underlying the impaired 2-Fly-induced relaxation of aortas from SHR, we evaluated 2-Fly-induced relaxation under various conditions (Fig. 2 and Table 1). Compared with the initial preparation (Fig. 1A), 2-Fly-induced relaxation was considerably reduced by treatment with PAR2 antagonist (Fig. 2A) or endothelial denudation (Fig. 2B). In addition, differences in relaxation between the SHR and WKY groups was completely abolished under these conditions. NOS inhibition by L-NNA also considerably reduced 2-Fly-induced relaxation in both the groups, although some relaxation was observed in the WKY group (Fig. 2C). Treatment with the Akt inhibitor did not affect 2-Fly-induced relaxation in both groups (Fig. 2D).

\section{DISCUSSION}

Chronic hypertension leads to vascular dysfunction within systemic organs, but the pathophysiological mechanisms underlying these effects remain unclear. Impaired PAR2-induced vascular relaxation was reportedly observed in rats with metabolic syndrome..$^{9-12)}$ However, whether chronic hypertension affects PAR2-induced relaxation remains unclear. In this study, we focused on vasorelaxant responses induced by the PAR2 agonist 2-Fly, ACh, and SNP in aortas isolated from aged SHRs and WKY rats.

Reportedly, in vivo and in vitro studies suggested that PAR2-mediated vasorelaxations are associated with endothelial activation and partly through the release of NO. ${ }^{9,23,24)}$ Here, 2-Fly-induced aortic relaxation significantly decreased in the SHR group compared with that in the WKY group. Furthermore, 2-Fly-induced relaxation was considerably decreased by treatment with the PAR2 antagonist and was abolished by endothelial denudation. These results suggested that 2-Flyinduced aortic relaxation was dependent on PAR2 activation in the endothelium. In the present study, NOS inhibition by L-NNA considerably decreased 2-Fly-induced relaxation in both the groups, although small relaxation was observed in the WKY group. Moreover, endothelium-dependent relaxation of aortas by another endothelium-dependent dilator ACh significantly decreased in the SHR group, whereas NO donor-induced endothelium-independent relaxation was similar between the SHR and WKY groups. These results suggested that impaired PAR2-induced aortic relaxation might be attributed to reduced NO bioavailability (i.e., reduction of NO production/release or rapid destruction of NO) rather than impairment of NO sensitivity in vascular smooth muscle. The activations of eNOS upon various endothelial stimulators including PAR2 ligand were attributable to not only phosphorylate eNOS by various kinases but also associate with $\mathrm{Ca}^{2+}$ / calmodulin..$^{9,25-30)}$ To investigate relationship between impaired 2-Fly-induced relaxation and Akt because PAR2 activation increased Akt activity in various cells and Akt activation could modulate vascular tone including eNOS activation, ${ }^{28-32}$ ) we performed concentration-response curves for 2-Fly under Akt inhibition. Unexpectedly, treatment with the Akt inhibitor did not affect 2-Fly-induced relaxation in both the groups, suggesting that the impaired 2-Fly-induced aortic relaxation was not due to activation of Akt. Moreover, no alterations of eNOS expression and phosphorylation at Ser1177 of eNOS treated with and without 2-Fly $\left(10^{-6} \mathrm{M}\right.$ for $\left.10 \mathrm{~min}\right)$ were seen in aortas of these aged SHR and WKY (data not shown). Although NO is a major endothelium-derived relaxing factor in the aorta, ${ }^{26,27)}$ there is a possibility that other vasorelaxant mediators in addition to NO may also partly participate in the 2-Fly-induced relaxation including prostanoids. ${ }^{33,34)}$ At present, we cannot rule out the possibility of altered signaling of other vascular tone modulators induced by PAR2 activation. Future studies should investigate the mechanisms underlying impaired 2-Fly-induced relaxation of aortas of aged SHR.

Several reports suggested that the association between PAR2 expression and functional properties. ${ }^{9)}$ For example, Kwapiszewska et al. demonstrated that increased expressions of PAR2 was observed in lung from idiopathic pulmonary hypertensive patients (vs. donor). ${ }^{35)}$ Aman et al. found that increased expression of PAR2 protein in aorta from female SHR at $10 \pm 1$ weeks old (vs. age-matched female WKY) and responded to trypsin. ${ }^{36)}$ Sobey et al. found that activation of PAR2 caused NO-mediated relaxation and such relaxation preserved/augmented in basilar arteries of SHR ( vs. WKY) at 6 to 11 months old and no apparent difference of PAR2 immu- 
noreactivity in endothelial and smooth muscle cells between SHR and WKY. ${ }^{37)}$ Using metabolic syndrome model SHRSP. ZF rat, which strain was established by crossbreeding strokeprone spontaneously hypertensive rats (SHRSP) and Zucker fatty rats $(\mathrm{ZF}),{ }^{38)}$ Kagota et al. investigated responsiveness to PAR2 ligands ${ }^{11,12)}$ and the relationship among metabolic parameters, PAR2-mediated relaxations, and PAR2 expressions in detail. ${ }^{9,10)}$ They clearly demonstrated that 1) systolic blood pressure correlated with PAR2-induced relaxation, but not with expression of mRNA for PAR2, 2) blood triglyceride and glucose, and body weight were not correlated with either PAR2-induced relaxation or PAR2 expression, and 3) increased levels of thiobarbituric acid, a marker of oxidative stress, negatively correlated with PAR2-induced relaxation and mRNA expression for PAR2. ${ }^{10)}$ In our unpublished observation, the protein expression of PAR2 in whole tissue homogenates of aorta (viz. including endothelial and smooth muscle cells) from rats (at $52 \pm 1$ weeks old) upon stimulated with or without 2-Fly $\left(10^{-6} \mathrm{M}\right.$ for $\left.10 \mathrm{~min}\right)$ did not alter among 2-Fly-unstimulated and -stimulated SHR and WKY groups. However, in the present study, we can only report the impaired PAR2-induced relaxation was seen in aortas from aged SHR compared to age-matched WKY. However, further study is required on time-dependent alterations in endothelial function, PAR2-mediated relaxation, PAR2 expression in not only endothelial cells but also smooth muscle cells, and putative causative factors (e.g., oxidative stress) in this.

In conclusion, our study findings demonstrated that PAR2induced relaxations of aortas of aged SHR was impaired, and this impaired aortic relaxation may be attributed to decreased NO bioavailability rather than altered NO sensitivity unrelated to the Akt activity. We believe that our present findings should stimulate further interest in the vascular responsiveness to PAR2 activation as a potential therapeutic target to improve chronic hypertension-associated vasculopathies.

Acknowledgments We thank Mr. K. Takayanagi, Mrs. M. Kojima, Mr. M. Ichikawa, Mrs. M. Iwasaki, Mr. S. Ohira, Mrs. K. Taguchi, Mr. S. Noguchi, Mrs. K. Hatano, and Mrs. Y. Higashida for technical assistance. This study was supported in part by JSPS KAKENHI Grants JP17K07975 to K.T. and JP15K07975 to T.K.

Conflict of Interest The authors declare no conflict of interest.

\section{REFERENCES}

1) Buford TW. Hypertension and aging. Ageing Res. Rev., 26, 96-111 (2016).

2) Vanhoutte PM, Zhao Y, Xu A, Leung SW. Thirty years of saying NO: sources, fate, actions, and misfortunes of the endotheliumderived vasodilator mediator. Circ. Res., 119, 375-396 (2016).

3) Daiber A, Steven S, Weber A, Shuvaev VV, Muzykantov VR, Laher I, Li H, Lamas S, Munzel T. Targeting vascular (endothelial) dysfunction. Br. J. Pharmacol., 174, 1591-1619 (2017).

4) Matsumoto $\mathrm{T}$, Goulopoulou S, Taguchi K, Tostes RC, Kobayashi T. Constrictor prostanoids and uridine adenosine tetraphosphate: vascular mediators and therapeutic targets in hypertension and diabetes. Br. J. Pharmacol., 172, 3980-4001 (2015).

5) Soh UJ, Dores MR, Chen B, Trejo J. Signal transduction by protease-activated receptors. Br. J. Pharmacol., 160, 191-203 (2010).
6) Rothmeier AS, Ruf W. Protease-activated receptor 2 signaling in inflammation. Semin. Immunopathol., 34, 133-149 (2012).

7) Kagota S, Maruyama K, McGuire JJ. Characterization and functions of protease-activated receptor 2 in obesity, diabetes, and metabolic syndrome: a systematic review. Biomed. Res. Int., 2016, 3130496 (2016).

8) McGuire JJ. Proteinase-activated receptor 2 (PAR2): a challenging new target for treatment of vascular diseases. Curr. Pharm. Des., 10, 2769-2778 (2004).

9) Maruyama K, McGuire JJ, Kagota S. Progression of time-dependent changes to the mechanisms of vasodilation by protease-activated receptor 2 in metabolic syndrome. Biol. Pharm. Bull., 40, 2039-2044 (2017).

10) Maruyama K, Kagota S, McGuire JJ, Wakuda H, Yoshikawa N, Nakamura K, Shinozuka K. Age-related changes to vascular proteaseactivated receptor 2 in metabolic syndrome: a relationship between oxidative stress, receptor expression, and endothelium-dependent vasodilation. Can. J. Physiol. Pharmacol., 95, 356-364 (2017).

11) Maruyama K, Kagota S, McGuire JJ, Wakuda H, Yoshikawa N, Nakamura K, Shinozuka K. Enhanced nitric oxide synthase activation via protease-activated receptor 2 is involved in the preserved vasodilation in aortas from metabolic syndrome rats. J. Vasc. Res., 52, 232-243 (2015).

12) Kagota S, Maruyama K, Wakuda H, McGuire JJ, Yoshikawa N, Nakamura K, Shinozuka K. Disturbance of vasodilation via protease-activated receptor 2 in SHRSP.Z-Lepr fa/IzmDmcr rats with metabolic syndrome. Vascul. Pharmacol., 63, 46-54 (2014).

13) McGuire JJ, Hollenberg MD, Andrade-Gordon P, Triggle CR. Multiple mechanisms of vascular smooth muscle relaxation by the activation of protease-activated receptor 2 in mouse mesenteric arterioles. Br. J. Pharmacol., 135, 155-169 (2002).

14) Hamilton JR, Nguyen PB, Cocks TM. Atypical protease-activated receptor mediates endothelium-dependent relaxation of human coronary arteries. Circ. Res., 82, 1306-1311 (1998).

15) Hamilton JR, Frauman AG, Cocks TM. Increased expression of protease-activated receptor-2 (PAR2) and PAR4 in human coronary artery by inflammatory stimuli unveils endothelium-dependent relaxation to PAR2 and PAR4 agonists. Circ. Res., 89, 92-98 (2001).

16) Saifeddine M, Al-Ani B, Cheng C-H, Wang L, Hollenberg MD. Rat proteinase-activated receptor-2 (PAR2): cDNA sequence and activity of receptor-derived peptides in gastric and vascular tissue. $\mathrm{Br} . J$. Pharmacol., 118, 521-530 (1996).

17) Kagota S, Chia E, McGuire JJ. Preserved arterial vasodilatation via endothelial protease-activated receptor- 2 in obese type 2 diabetic mice. Br. J. Pharmacol., 164, 358-371 (2011).

18) Matsumoto $T$, Ishida $K$, Taguchi $K$, Kobayashi $T$, Kamata K. Mechanisms underlying enhanced vasorelaxant response to protease-activated receptor 2-activating peptide in type 2 diabetic GotoKakizaki rat mesenteric artery. Peptides, 30, 1729-1734 (2009).

19) McGuire JJ, Van Vliet BN, Gimenez J, King JC, Halfyard SJ. Persistence of PAR-2 vasodilation despite endothelial dysfunction in BPH/2 hypertensive mice. Pflugers Arch., 454, 535-543 (2007).

20) Watanabe S, Matsumoto T, Ando M, Kobayashi S, Iguchi M, Taguchi K, Kobayashi T. A comparative study of vasorelaxant effects of ATP, ADP, and adenosine on the superior mesenteric artery of SHR. Biol. Pharm. Bull., 39, 1374-1380 (2016a).

21) Watanabe S, Matsumoto T, Ando M, Kobayashi S, Iguchi M, Taguchi K, Kobayashi T. Effect of long-term diabetes on serotoninmediated contraction in carotid arteries from streptozotocin-induced diabetic male and female rats. Biol. Pharm. Bull., 39, 1723-1727 (2016b).

22) Matsumoto $T$, Watanabe S, Kobayashi S, Ando M, Taguchi K, Kobayashi T. Age-related reduction of contractile responses to urotensin ii is seen in aortas from wistar rats but not from type 2 diabetic Goto-Kakizaki rats. Rejuvenation Res., 20, 134-145 (2017). 23) Cicala C. Protease activated receptor 2 and the cardiovascular sys- 
tem. Br. J. Pharmacol., 135, 14-20 (2002).

24) Cicala C, Morello S, Vellecco V, Severino B, Sorrentino L, Cirino G. Basal nitric oxide modulates vascular effects of a peptide activating protease-activated receptor 2. Cardiovasc. Res., 60, 431-437 (2003).

25) Sessa WC. eNOS at a glance. J. Cell Sci., 117, 2427-2429 (2004).

26) Kobayashi T, Nemoto S, Ishida K, Taguchi K, Matsumoto T, Kamata $\mathrm{K}$. Involvement of CaM kinase II in the impairment of endothelial function and eNOS activity in aortas of type 2 diabetic rats. Clin. Sci., 123, 375-386 (2012).

27) Taguchi K, Morishige A, Matsumoto $T$, Kamata K, Kobayashi $T$. Enhanced estradiol-induced vasorelaxation in aortas from type 2 diabetic mice may reflect a compensatory role of p38 MAPK-mediated eNOS activation. Pflugers Arch., 464, 205-215 (2012).

28) Kobayashi T, Taguchi K, Yasuhiro T, Matsumoto T, Kamata K. Impairment of PI3-K/Akt pathway underlies attenuated endothelial function in aorta of type 2 diabetic mouse model. Hypertension, 44, 956-962 (2004).

29) Kobayashi T, Taguchi K, Nemoto $S$, Nogami T, Matsumoto T, Kamata $\mathrm{K}$. Activation of the PDK-1/Akt/eNOS pathway involved in aortic endothelial function differs between hyperinsulinemic and insulin-deficient diabetic rats. Am. J. Physiol. Heart Circ. Physiol., 297, 1767-1775 (2009).

30) Taguchi K, Matsumoto T, Kamata K, Kobayashi T. Akt/eNOS pathway activation in endothelium-dependent relaxation is preserved in aortas from female, but not from male, type 2 diabetic mice. Pharmacol. Res., 65, 56-65 (2012).

31) Rasmussen JG, Riis SE, Frobert O, Yang S, Kastrup J, Zachar V, Simonsen U, Fink T. Activation of protease-activated receptor 2 in- duces VEGF independently of HIF-1. PLOS ONE, 7, e46087 (2012).

32) Du C, Zhang T, Xiao X, Shi Y, Duan H, Ren Y. Protease-activated receptor-2 promotes kidney tubular epithelial inflammation by inhibiting autophagy via the PI3K/Akt/mTOR signaling pathway. Biochem. J., 474, 2733-2747 (2017).

33) Robin J, Kharbanda R, Mclean P, Campbell R, Vallance P. Proteaseactivated receptor 2-mediated vasodilatation in humans in vivo: role of nitric oxide and prostanoids. Circulation, 107, 954-959 (2003).

34) Roviezzo F, Bucci M, Brancaleone V, Di Lorenzo A, Geppetti P, Farneti S, Parente L, Lungarella G, Fiorucci S, Cirino G. Proteinase-activated receptor- 2 mediates arterial vasodilation in diabetes. Arterioscler. Thromb. Vasc. Biol., 25, 2349-2354 (2005).

35) Kwapiszewska G, Markart P, Dahal BK, Kojonazarov B, Marsh LM, Schermuly RT, Taube C, Meinhardt A, Ghofrani HA, Steinhoff M, Seeger W, Preissner KT, Olschewski A, Weissmann N, Wygrecka M. PAR-2 inhibition reverses experimental pulmonary hypertension. Circ. Res., 110, 1179-1191 (2012).

36) Aman M, Hirano M, Kanaide H, Hirano K. Upregulation of proteinase-activated receptor-2 and increased response to trypsin in endothelial cells after exposure to oxidative stress in rat aortas. $J$. Vasc. Res., 47, 494-506 (2010).

37) Sobey CG, Moffatt JD, Cocks TM, Kontos HA. Evidence for selective effects of chronic hypertension on cerebral artery vasodilatation to protease-activated receptor-2 activation. Stroke, 30, 1933-1940, discussion, 1941 (1999).

38) Hiraoka-Yamamoto J, Nara Y, Yasui N, Onobayashi Y, Tsuchikura S, Ikeda K. Establishment of a new animal model of metabolic syndrome: SHRSP fatty (fa/fa) rats. Clin. Exp. Pharmacol. Physiol., 31, 107-109 (2004). 